\title{
An Electrochemical Immunobiosensor for Direct Detection of Veterinary Drug Residues in Undiluted Complex Matrices
}

\author{
Sook Mei Khor,${ }^{\mathrm{a}}$ Guozhen Liu,${ }^{\mathrm{a}}$ Joshua R. Peterson, ${ }^{\mathrm{a}}$ Sridhar G. Iyengar,${ }^{\mathrm{b}}$ J. Justin Gooding*a \\ a School of Chemistry, The University of New South Wales, Sydney NSW 2052, Australia \\ b AgaMatrix, Inc. 10 Manor Parkway Salem, NH 03079, USA \\ *e-mail: justin.gooding@unsw.edu.au
}

Received: April 11, 2011

Accepted: May 3, 2011

\begin{abstract}
An electrochemical immunobiosensor is developed that allows the detection of small molecules, such as drugs, in undiluted complex samples, with no washing or rinsing steps via a displacement assay. This is achieved using an interface comprised of a mixed layer of oligo(phenylethynylene) molecular wire (MW), to allow electrochemical communication, and oligo(ethylene glycol) (OEG) to control the interaction of proteins and electroactive interferences with the electrode surface. The mixed layer is formed from in situ-generated aryl diazonium cations. To the distal end of the MW, a redox probe 1,1'-di(aminomethyl)ferrocene (FDMA) is attached followed by an epitope (the structural feature the antibody selectively recognizes) to which an antibody would bind. Association or dissociation of the antibody with the sensing interface causes a modulation of the ferrocene electrochemistry. Antibody complexed electrodes are exposed to samples containing spiked enrofloxacin (unbound target analyte), in milk and environmental water and interrogated using square wave voltammetry (SWV). The lowest detected concentration of free enrofloxacin was $10 \mathrm{pg} \mathrm{mL}^{-1}$ in phosphate buffer, $50 \mathrm{mM}, \mathrm{pH}$. For free enrofloxacin detection in undiluted complex matrices, by adding disodium EDTA $(50 \mathrm{mM})$, the recovery obtained was $94.1 \%$ in skim milk and $88.5 \%$ in stream water, respectively as compared to clean phosphate buffer. The immunobiosensor response time was 10 15 minutes. The sensor performance in milk was shown to be superior to a standard method based on Liquid Chromatography Mass Spectroscopy (LC-MS/MS).
\end{abstract}

Keywords: Electrochemical immunobiosensor, Veterinary drug residue, Complex matrices, Small molecules, User intervention free

DOI: 10.1002/elan.201100205

Supporting information for this article is available on the WWW under http://dx.doi.org/10.1002/elan.201100205.

\section{Introduction}

The selective detection of small molecules $(<1 \mathrm{kDa})$ such as antibiotic residues [1] and pesticides [2], is an important but challenging task for their routine monitoring in complex samples such as food, environmental waters and biological fluids. This challenge is exacerbated for portable analytical devices where it is desirable for the method to be user intervention free; that is it requires no washing or rinsing steps. There is considerable interest in electrochemical immunoassays for small molecules [3-7]. However most approaches require the user to either add reagents for signal transduction and/or multiple washing or rinsing steps are needed $[4,6,7]$. To overcome the need for the user to do anything other than place the immunosensor into the sample, we recently reported a new electrochemical immunosensing concept $[8,9]$. In this concept a glassy carbon electrode is modified with a mixed layer of molecular wire (MW) [10] and oligo(ethylene glycol) (OEG) [11] (Scheme 1). To the end of the MW is attached a redox probe 1,1'-di(aminomethyl)ferrocene (FDMA) and an epitope (ciprofloxacin) (Figure 1a) to which an antibody (monoclonal anti-enrofloxacin IgM antibody) is specifically complexed. Complexation of the antibody immerses the redox probe in a protein environment and causes a significant attenuation of the current derived from the ferrocene moiety. The attenuation of current upon antibody binding is attributed to the restriction of counter ions accessing the redox probe to balance the charge associated with electron transfer, thereby hindering the electron transfer. Since there is no labelling of antibody or antigen required, the biosensor is used simply by placing it into a sample. When placed in a sample containing the target analyte (free enrofloxacin, Figure 1b), transduction is achieved by antibody dissociating from the electrode surface due to the competition for the antibody between the surface bound epitope and the analyte. In other words, the current increases upon dissociation of the antibody from the surface bound epitope; an event motivated by the presence of the analyte free in solution (small unbound molecules). This type of assay is referred to as a displacement assay (Scheme 1). The system has yet to be applied to analytes in complex samples. The purpose of this paper is to demonstrate the ability of this 